\title{
Unusual Origin of the Arteries in the Carotid Triangle of the Neck: A Case Report and Literature Review
}

\author{
Rao Mohandas K.G. ${ }^{*}, 1$ and Rao Asutosh S. ${ }^{2}$ \\ ${ }^{I}$ Department of Anatomy, ${ }^{2}$ Department of Orthopedics, Faculty of Medicine, AIMST University, Semeling, Kedah, \\ Malaysia
}

\begin{abstract}
Though the variations in the carotid arteries and their branches are common in the carotid triangle, it is important for the clinicians, surgeons and the radiologists to be aware of all possible variations of these arteries. A case of abnormal origin of 2 important arteries encountered during routine dissection of carotid triangle of the neck is reported. The superior thyroid artery that normally originates form the external carotid was seen to originate from the common carotid artery and the superior laryngeal artery was arising from the external carotid artery instead of the superior thyroid artery. Further, a detailed literature review was done and the surgical, radiological and clinical importance of the case was discussed.
\end{abstract}

Keywords: Superior thyroid artery, superior laryngeal artery, external carotid artery, common carotid artery, carotid triangle, arterial variations in the neck.

\section{INTRODUCTION}

Knowledge of variations of the common carotid and external carotid arteries and their branches are of great importance during radiological examinations, intervention radiology and surgery [1]. Normally, the common carotid artery (CCA) ends in 2 terminal branches, i.e. external and internal carotid, at the level of upper border of thyroid cartilage in the carotid triangle.

\section{The Normal Course}

The external carotid artery (ECA) runs upwards after its origin from the CCA and passes deep to the posterior belly of the digastric muscle to enter the digastric triangle and later into the parotid gland. In the carotid triangle, it normally gives five branches namely the superior thyroid, lingual, facial, ascending pharyngeal and occipital arteries (Fig. 1).

The superior thyroid artery (STA), a branch of the ECA, normally runs downwards from its origin and gives rise to superior laryngeal artery (SLA) which pierces the thyrohyoid membrane along with internal laryngeal nerve (Fig. 1) [2].

\section{CASE REPORT}

During routine dissection of a 60 -year-old Indian male cadaver (preserved by formalin fixation method) used for teaching of medical undergraduates in the Department of Anatomy, Faculty of Medicine, AIMST University, Malaysia, we encountered variations in the origin of two arteries in the left carotid triangle of the neck.

The common carotid artery bifurcated at its normal level of the superior border of thyroid cartilage. The superior thyroid artery (STA) was arising from the common carotid artery $(\mathrm{CCA})$ about $1 \mathrm{~cm}$ proximal to its bifurcation. This

*Address correspondence to this author at the Faculty of Medicine, AIMST University, Semeling, Bedong- 08100, Kedah Darul Aman, Malaysia; Tel: +60164977261; Fax: 604-4298009; E-mail: mohandaskg@gmail.com

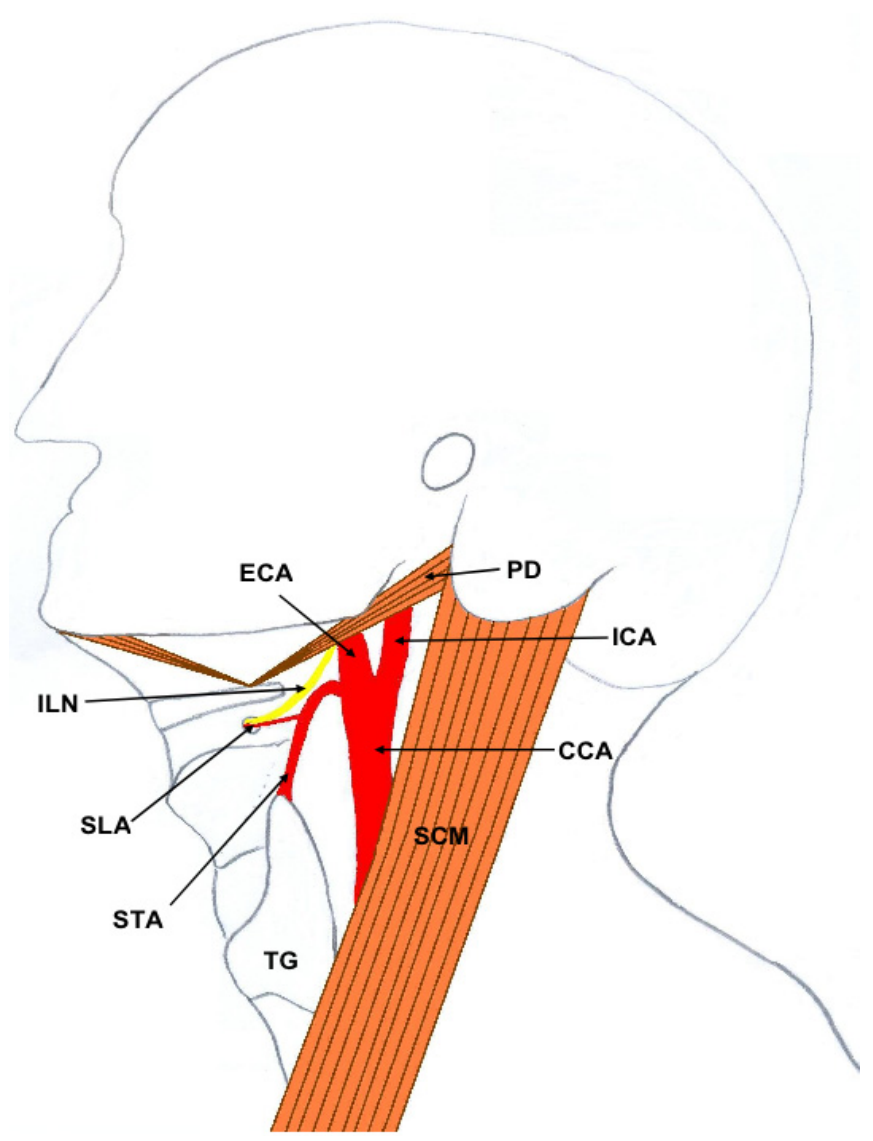

Fig. (1). Schematic diagram of the carotid triangle of the neck illustrating the normal course of carotid arteries and origin of superior thyroid artery (STA). Note that the superior thyroid artery (STA) is arising from the external carotid artery (ECA) and superior laryngeal artery (SLA) is a branch of superior thyroid artery (STA). (CCA- Common carotid artery, ICA- Internal carotid artery, TG- Thyroid gland, SCM- Sternocleidomastoid, PDPosterior belly of digastric, ILN- Internal laryngeal nerve). 
artery then passed downwards towards the apex of the thyroid lobe. Although STA was arising abnormally from the CCA, it was still accompanied by the external laryngeal nerve.

The superior laryngeal artery (SLA) which normally is a branch of STA was arising from the external carotid artery (ECA) almost from the same point where STA would normally arise. The SLA was accompanied by the internal laryngeal nerve and both these structures together pierced the thyrohyoid membrane.

The origin and course of lingual, ascending pharyngeal, occipital, posterior auricular and terminal branches of external carotid artery were normal. Similarly, there were no variations among these arteries on the right side of the neck (Figs. 2-4).

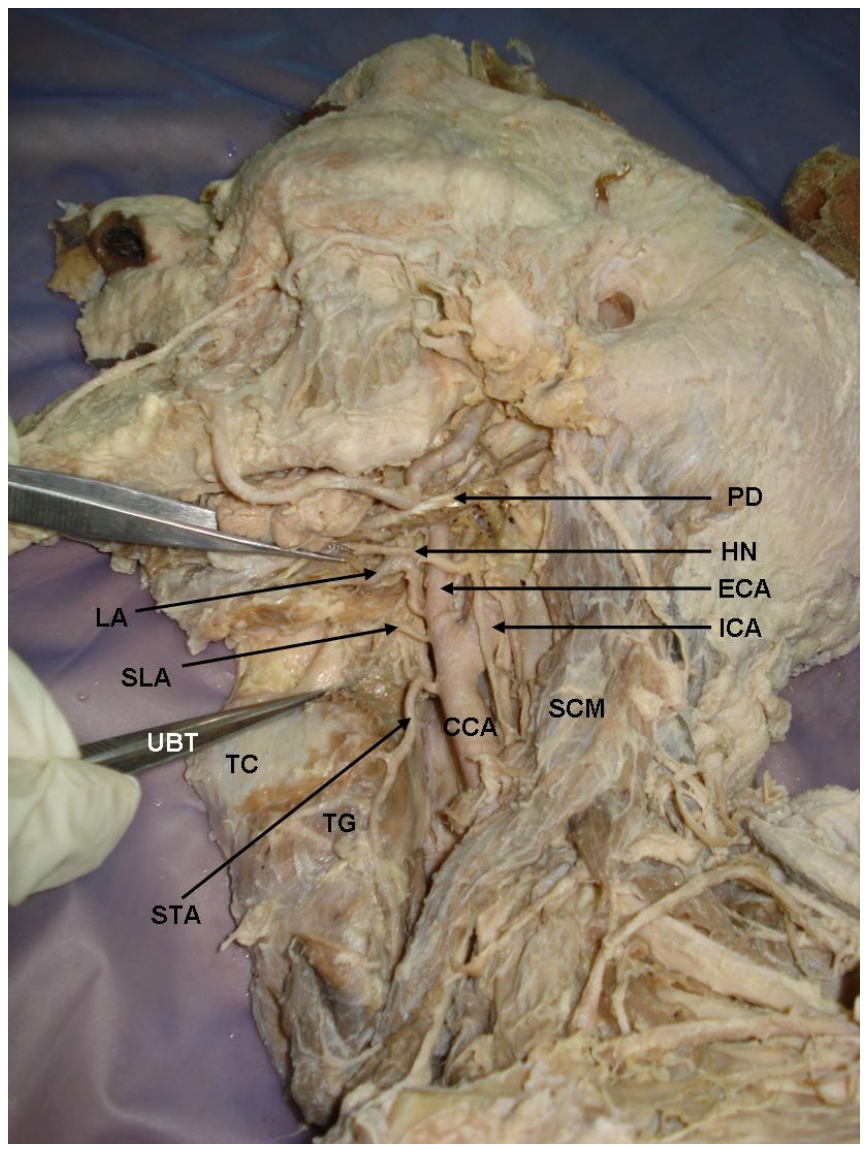

Fig. (2). Dissection of the carotid triangle of the neck illustrating the carotid arteries and their branches. Note that superior thyroid artery (STA) is arising from the common carotid artery (CCA) instead of external carotid artery (ECA). Similarly, superior laryngeal artery (SLA) is originating from the external carotid artery (ECA) instead of superior thyroid artery (STA). The position of the forceps indicates the upper border of thyroid cartilage (UBT) which is the normal level of common carotid artery bifurcation. (LA- Lingual artery, TC- Thyroid cartilage, TG- Thyroid gland, SCM- Sternocleidomastoid, ICA- Internal carotid artery, HNHypoglossal nerve, PD- Posterior belly of digastric).

\section{DISCUSSION}

It is universally accepted that the common carotid artery divides into the external and internal carotid arteries at the

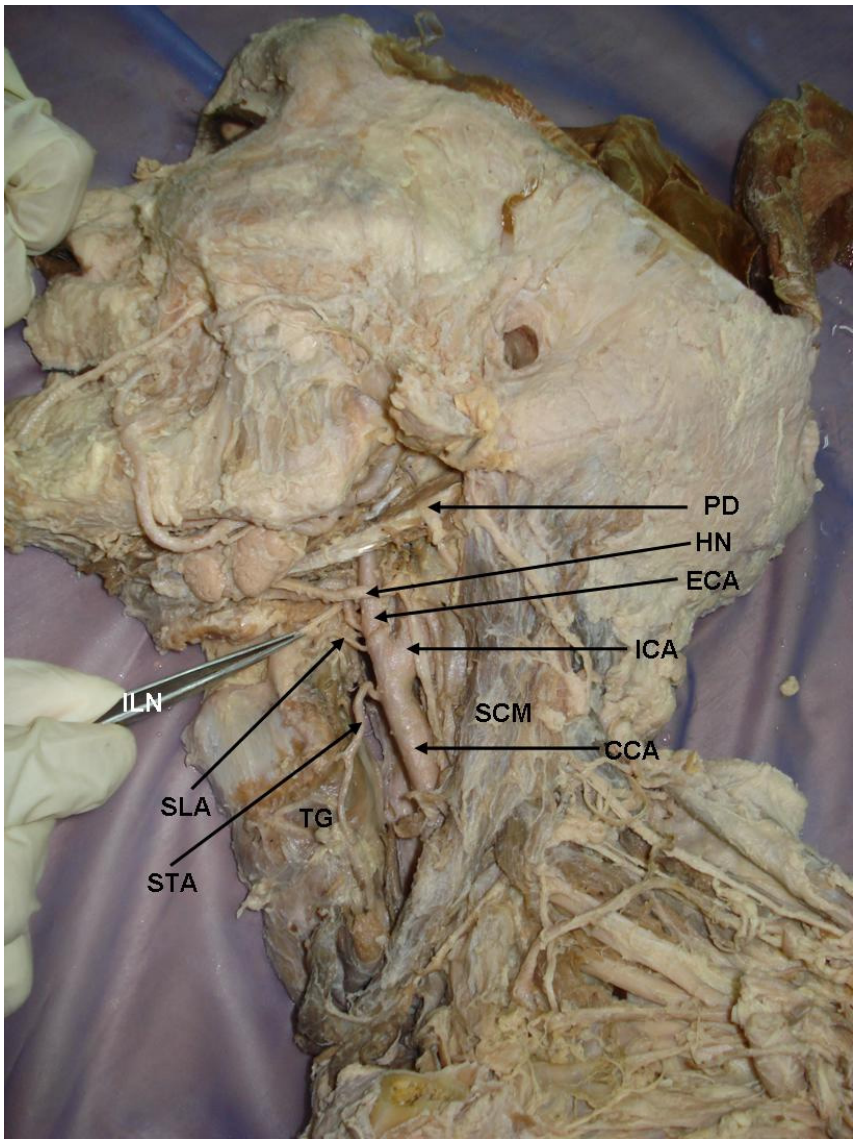

Fig. (3). Dissection of the carotid triangle of the neck illustrating the carotid arteries and their branches. Note that superior thyroid artery (STA) is arising from the common carotid artery (CCA) instead of external carotid artery (ECA). Similarly, superior laryngeal artery (SLA) is originating from the external carotid artery (ECA) instead of superior thyroid artery (STA). The forceps is showing the internal laryngeal nerve (ILN) which accompanies the superior laryngeal artery (SLA) to pierce the thyrohyoid membrane. (TG- Thyroid gland, SCM- Sternocleidomastoid, ICAInternal carotid artery, HN- Hypoglossal nerve, PD- Posterior belly of digastric).

level of the superior border of the thyroid cartilage. Ilic found this to be true in $58 \%$ of cases, Espalieu et al. in $65 \%$ of cases, and Von Poisel and Golth in $67 \%$ of cases [3]. Lucev et al. conducted a study to assess the normal level of carotid bifurcation and concluded that in $50 \%$ of cases the level of the bifurcation corresponded to the superior border of the thyroid cartilage. Carotid bifurcation occurred in $25 \%$ at the level of the inferior border of the hyoid bone. A higher level of bifurcation, opposite the superior border of the hyoid bone was found in $12.5 \%$. Bifurcation appeared at the level of the inferior border of the thyroid cartilage in $12.5 \%$ [3]. In this cadaver the CCA bifurcation was found to be placed normally at the level of the superior border of the thyroid cartilage.

Variations in the branches of ECA are very common. Because of high probability of variations in the arteries of carotid and digastric triangles, a number of studies have been carried out on these arteries and a wide range of variations have been reported. Recently, Vázquez et al. have dissected 


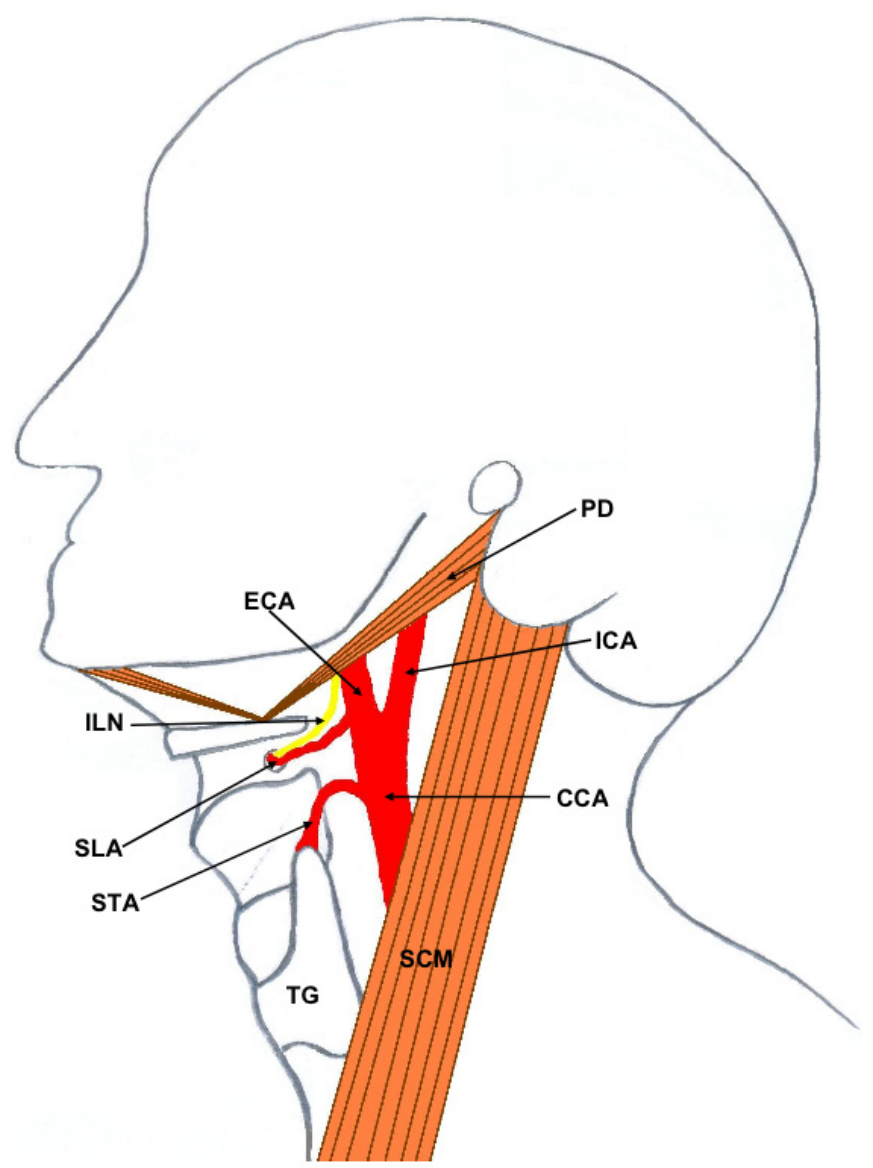

Fig. (4). Schematic diagram of the carotid triangle of the neck illustrating the variant origin of the superior thyroid artery (STA) and superior laryngeal artery (SLA). Note that superior thyroid artery (STA) is arising from the common carotid artery (CCA) instead of external carotid artery (ECA). Similarly, superior laryngeal artery (SLA) is originating from the external carotid artery (ECA) instead of superior thyroid artery (STA). Internal laryngeal nerve (ILN) which accompanies the superior laryngeal artery (SLA) to pierce the thyrohyoid membrane is also shown. (ICA- Internal carotid artery, TG- Thyroid gland, SCMSternocleidomastoid, PD- Posterior belly of digastric).

330 human hemi-necks and studied the variations of superior thyroid and superior laryngeal arteries [4]. They have reported 4 different origins for the STA, the most frequent type being from the carotid bifurcation (49\%). Similarly, they have reported 4 different origins for the SLA and most frequent type is one in which the artery arose from STA (78\%) [4]. Although, standard literature [2] observes that the STA as a branch of the ECA, Lucev et al. have reported that STA arises more often from the common carotid artery (47.5\% of cases) than from the external carotid artery $(30 \%$ of cases). In about $22.5 \%$ of cases STA was reported to be originating from the carotid bifurcation [3]. Similar to this observation by Lucev et al., the STA in this cadaver was found to be arising from the CCA.

In a study on front branches of ECA, Ozgur et al. have observed that in $90 \%$ cases, the 3 front branches i.e., the superior thyroid, facial \& lingual arose separately from ECA and in all cases STA originated from the ECA about $3.9 \mathrm{~mm}$ distal to carotid bifurcation [5]. When it arose from the external carotid artery, the distance of origin from the bifurcation ranged from 2 to $10.5 \mathrm{~mm}$. The distance of origin from the common carotid artery to the bifurcation also ranged from 2 to $10.7 \mathrm{~mm}$ [3]. Lemaire et al. reported the origin of the superior thyroid artery from a common thyrolingual trunk $30 \mathrm{~mm}$ below the bifurcation of the common carotid artery [6].

A study conducted on the distribution of the ECA trunks in human fetuses showed thyrolingual trunk in $2.5 \%$ and a thyrolinguofacial trunk in $2.5 \%$ of the cases [7].

A Meta analysis conducted by Toni et al. to investigate whether the presence, numerical variations and site of origin of the STA are influenced by the ethnic group and gender has revealed a higher frequency of its origin from the external carotid artery in Caucasoids than in East Asians. No gender differences were found in East Asians. An equal probability of either asymmetrical or symmetrical origin on the two sides of the neck for STA was found in East Asians. In contrast, asymmetry of caliber for STA was found in European Caucasoids [8].

A case of absence of left STA has also been reported [9]. There are also case reports of origin of STA from the common carotid artery [9-14]. However in most of these cases, the origin of STA from CCA was associated with a high bifurcation of the CCA. In the present case, the STA is arising from the CCA that is terminating at the normal level i.e. at the upper border of thyroid cartilage.

One case of a double SLA - one from STA and the other from the ECA has been reported [15]. Superior laryngeal artery arising from the ECA replacing the site of origin of STA is being reported for the first time.

\section{Clinical Importance}

Profound knowledge of the anatomical characteristics and variations of the carotid artery, such as its branching pattern and position is essential to avoid complications with catheter insertion of carotid arteries in various procedures [14]. Micrometric values of structures in the neck are accepted as important orientation points during surgical intervention [5], hence it is essential to know the possible variations and minor details of the branches of ECA in the neck. Variations in the origin of STA and SLA from the carotid arterial tree and the similarity of their diameters would lead to a significant possibility of wrong identification during surgery [4].

It is also important to know of possible variations in patterns of origin, courses, and branching pattern of the STA during surgical procedures in the neck region, such as during emergency cricothyroidotomy, radical neck dissection, carotid catheterization, reconstruction of aneurysm and carotid endarterectomy [10]. Lack of experience with possible variations could lead to fatal errors if one blood vessel should be mistaken for another [3]. Knowledge of the surgical anatomy of the STA ensures maintaining a bloodless surgical field during major radical neck dissection surgeries to minimize postoperative complications [10]. The superior thyroid artery also takes part in the formation of important collateral circulation between the external carotid arteries that become important in cases of occlusion of unilateral common carotid artery $[16,17]$. 
From the above literature review, it is evident that there is a possibility of wide range of variations in the STA, SLA and other branches of ECA. Abnormalities observed in our case, adds to the long list of known variations of these arteries and could help avoid serious implications during radiological examinations, interventions, ultrasound examination, exploration of the neck, thyroid and parathyroid surgery, tracheotomy, surgery of the larynx, pharynx and upper esophagus and microvascular surgeries $[3,18]$.

\section{REFERENCES}

[1] Gluncic V, Petanjek Z, Marusic A, Gluncic I. High bifurcation of common carotid artery, anomalous origin of ascending pharyngeal artery and anomalous branching pattern of external carotid artery. Surg Radiol Anat 2001; 23:123-5.

[2] Standring S, Ellis H, Healy JC, Johnson D, Williams A, Collins P. From Gray's anatomy: the anatomical basis of clinical practice. $39^{\text {th }}$ ed. London: Elsevier, Churchill Liwingstone 2005; pp. 543-7.

[3] Lucev N, Bobinac D, Maric I, Drescik I. Variations of the great arteries in the carotid triangle. Otolaryngol Head Neck Surg 2000; 122: 590-1.

[4] Vázquez T, Cobiella R, Maranillo E, et al. Anatomical variations of the superior thyroid and superior laryngeal arteries. Head Neck 2009; 31: 1078-85.

[5] Ozgur Z, Govsa F, Ozgur T. Assessment of origin characteristics of the front branches of the external carotid artery. J Craniofac Surg 2008; 19:1159-66.

[6] Lemaire V, Jacquemin G, Medot M, Fissette J. Thyrolingual trunk arising from the common carotid artery: a case report. Surg Radiol Anat 2001; 23:135-7.

[7] Zümre O, Salbacak A, Ciçekcibaşi AE, Tuncer I, Seker M. Investigation of the bifurcation level of the common carotid artery and variations of the branches of the external carotid artery in human fetuses. Ann Anat 2005; 187: 361-9.

[8] Toni R, Casa DC, Castorina S, et al. A meta-analysis of superior thyroid artery variations in different human groups and their clinical implications. Ann Anat 2004; 186: 255-62.

[9] Moriggl B, Sturm W. Absence of three regular thyroid arteries replaced by an unusual lowest thyroid artery (A. thyroidea ima): a case report. Surg Radiol Anat 1996;18:147-50.

[10] Ozgur Z, Govsa F, Celik S, Ozgur T. Clinically relevant variations of the superior thyroid artery: an anatomic guide for surgical neck dissection. Surg Radiol Anat 2009; 31:151-9.

[11] Klosek SK, Rungruang T. Topography of carotid bifurcation: considerations for neck examination. Surg Radiol Anat 2008; 30 383-7.

[12] Lo A, Oehley M, Bartlett A, Adams D, Blyth P, Al-Ali S. Anatomical variations of the common carotid artery bifurcation. ANZ J Surg 2006; 76: 970-2.

[13] Issing PR, Kempf HG, Lenarz T. A clinically relevant variation of the superior thyroid artery. Laryngorhinootologie 1994; 73: 536-7.

[14] Chitra R. Trifurcation of the right common carotid artery. Indian J Plast Surg 2008; 41: 85-8.

[15] Satheesha NB, Soumya KV. Neurovascular variations in the carotid triangle. Int J Anat Variations 2008; 1: 17-8.

[16] Macchi C, Catini C. The anatomy and clinical importance of the collateral circle between the external carotid arteries through an anastomosis between the superior thyroid arteries. Ital J Anat Embryol 1993; 98:197-205.

[17] Macchi C, Catini C. Clinical importance of the supra-isthmic anastomosis between the superior thyroid arteries in six cases of occlusion of the common carotid artery. Surg Radiol Anat 1995; 17: 65-9

[18] Espalieu P, Cottier M, Relave M, Youvarlakis P, Cuilleret J. Radioanatomic study of the carotid axis with regard to the implantation of microsurgical vascular anastomoses. Surg Radiol Anat 1986; 8: $257-63$. 